\title{
Vitamin E Gamma-tocopherol Reduces Airway Neutrophil Recruitment after Inhaled Endotoxin Challenge in Rats and in Healthy Volunteers
}

\author{
Michelle L Hernandez, MD ${ }^{\mathrm{a}}$, James G. Wagner, $\mathbf{P h D}^{\mathrm{b}}$, Aline Kala, $\mathbf{R N}^{\mathrm{a}}$, Katherine Mills, $\mathbf{B A}^{\mathrm{a}}$, \\ Heather B Wells, BS ${ }^{a}$, Neil E Alexis, PhD $^{a}$, John C Lay, PhD $^{a}$, Qing Jiang, PhD $^{\mathrm{c}}$, Hongtao \\ Zhang $^{d}$, Haibo Zhou, PhD ${ }^{\mathrm{a}, \mathrm{d}}$, and David B Peden, MD, MS ${ }^{\mathrm{a}}$ \\ ${ }^{a}$ Center for Environmental Medicine, Asthma, \& Lung Biology, The University of North Carolina at \\ Chapel Hill, Chapel Hill, NC

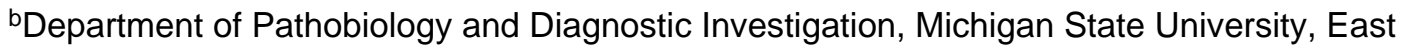 \\ Lansing, MI \\ 'Department of Nutrition Science, College of Health and Human Sciences, Purdue University, \\ West Lafayette, IN \\ dDepartment of Biostatistics, Gillings School of Global Public Health, The University of North \\ Carolina at Chapel Hill, Chapel Hill, NC
}

\begin{abstract}
Rationale-Epidemiologic studies suggest that dietary vitamin $\mathrm{E}$ is an important candidate intervention for asthma. Our group has shown that daily consumption of vitamin E (gamma tocopherol, $\gamma \mathrm{T}$ ) has anti-inflammatory actions in both rodent and human phase I studies. The objective of this study was to test whether $\gamma \mathrm{T}$ supplementation could mitigate a model of neutrophilic airway inflammation in rats and in healthy human volunteers.
\end{abstract}

Methods-F344/N rats were randomized to oral gavage with $\gamma \mathrm{T}$ versus placebo, followed by intranasal LPS (20 ug) challenge. Bronchoalveolar lavage fluid and lung histology were used to assess airway neutrophil recruitment. In a phase IIa clinical study, 13 nonasthmatic subjects completed a double-blinded, placebo controlled crossover study where they consumed either a $\gamma \mathrm{T}$ enriched capsule or a sunflower oil placebo capsule. After 7 days of daily supplementation, they underwent an inhaled LPS challenge. Induced sputum was assessed for neutrophils 6 hours after inhaled LPS. The effect of $\gamma \mathrm{T}$ compared to placebo on airway neutrophils post-LPS was compared using a repeated measures analysis of variance.

Results-In rats, oral $\gamma \mathrm{T}$ supplementation significantly reduced tissue infiltration $(\mathrm{p}<0.05)$ and accumulation of airway neutrophils $(\mathrm{p}<0.05)$ that are elicited by intranasal LPS challenge

\footnotetext{
(c) 2013 Elsevier Inc. All rights reserved.

Corresponding Author: Michelle L. Hernandez, MD, Center for Environmental Medicine, Asthma and Lung Biology, 104 Mason Farm Road, UNC School of Medicine, Chapel Hill, NC 27516, Michelle_Hernandez@med.unc.edu, phone 919-843-5383, fax 919-966-9863.

Publisher's Disclaimer: This is a PDF file of an unedited manuscript that has been accepted for publication. As a service to our customers we are providing this early version of the manuscript. The manuscript will undergo copyediting, typesetting, and review of the resulting proof before it is published in its final citable form. Please note that during the production process errors may be discovered which could affect the content, and all legal disclaimers that apply to the journal pertain.

Disclosures: D. B. Peden has been a consultant to GlaxoSmithKline and Aquinox Pharmaceuticals and a contract with MedImmune. The rest of the authors have declared that they have no conflicts of interest.
} 
compared to control rats. In human volunteers, $\gamma \mathrm{T}$ treatment significantly decreased induced sputum neutrophils ( $\mathrm{p}=0.03$ ) compared to placebo.

Conclusion-Oral supplementation with $\gamma \mathrm{T}$ reduced airway neutrophil recruitment in both rat and human models of inhaled LPS challenge. These results suggest that $\gamma \mathrm{T}$ is a potential therapeutic candidate for prevention or treatment of neutrophilic airway inflammation in diseased populations.

\section{Keywords}

Vitamin E; gamma-tocopherol; Endotoxin; Eosinophil; Neutrophil; Induced Sputum; Oxidative Stress; Nitrosative Stress; Rat; LPS

\section{Introduction}

Asthma is the leading cause of hospitalization in children and is a significant cause for morbidity in adults, accounting for 5,000 deaths per year in the U.S. The majority of asthma exacerbations are triggered by viral infections, pollutants which can injure or activate airway epithelial cells, or bioaerosols which likely activate airway monocytes and macrophages [1, 2]. Among environmental causes of asthma exacerbation, endotoxin (LPS) present in airborne particles is one of the most significant causes of acute disease in persons with established asthma [3].

LPS is a prototypic pathogen associated molecular pattern moiety which activates airway monocytes and macrophages through interaction with cell surface receptors CD14 and TLR4. Inhalation challenge studies of human volunteers demonstrate that LPS induces increased airway neutrophils (PMNs), increased pro-inflammatory cytokine levels (TNF-a, IL-6, IL-1 $\beta$ ), increased expression of monocyte or macrophage CD14 and TLR4, and increased oxidative stress, thus serving as a model of a neutrophilic asthma exacerbation[47]. Clinically, neutrophilic airway inflammation is difficult to treat, as neutrophils are relatively corticosteroid resistant [8], and treatment options are limited. Inhaled LPS challenge provides a mechanism to study neutrophil-predominant airways inflammation in early phase clinical studies prior to enrolling diseased populations.

Previous data from our laboratory suggests that gamma tocopherol $(\gamma \mathrm{T})$, a member of the vitamin $\mathrm{E}$ family, has novel therapeutic potential to mitigate LPS-induced inflammation. For example, $\gamma \mathrm{T}$ inhibited the production of prostaglandin E2 and the neutrophil chemoattractant leukotriene B4, as well as the production of pro-inflammatory cytokines such as TNFa and IL-6 after irritant challenge in animals [9]. The primary metabolite of $\gamma \mathrm{T}, 2,7,8$ trimethyl-2S-( $\gamma$-carboxyethyl)-6-hydroxychromane ( $\gamma$-CEHC), exhibited anti-inflammatory activity using in vivo rodent models of neutrophilic inflammation, as well as with LPS challenge of a macrophage cell line [10]. Neither $\gamma$ T nor $\gamma$-CEHC interfere with LPS binding to the TLR4 receptor, as neither had any effect on LPS-stimulated COX-2 expression in macrophages $[10,11]$. We also demonstrated that $\gamma$-CEHC inhibits reactive oxygen species generation by PMA-stimulated peripheral blood mononuclear cells [12], and that $\gamma$-CEHC inhibited LPS-induced NF- $\kappa \mathrm{B}$ activation by interfering with I $\kappa \mathrm{B} a$ degradation. Furthermore, after subjects consumed daily capsules of an oral $\gamma \mathrm{T}$-enriched supplement for 1 week as part of a phase I dosing study, ex-vivo macrophages stimulated with LPS had depressed production of the pro-inflammatory cytokines IL-1 $\beta$, TNF- $\alpha$, and IL-6 [12]. Taken together, these data suggest that supplementation with $\gamma$ T may be efficacious against LPS-induced inflammation.

In the present study, we first performed a pre-clinical study in rats to determine if oral gavage with $\gamma$ T could decrease airway neutrophil recruitment after inhaled LPS challenge. 
Based on the positive outcome of this initial study, we proceeded with a randomized double blinded, placebo controlled crossover study to test if oral $\gamma \mathrm{T}$ supplementation could suppress airway inflammatory responses after inhaled LPS challenge in healthy volunteers.

\section{Materials \& Methods ANIMAL STUDIES}

Animals-Male F344/N rats 9-10 weeks of age, were obtained from Harlan Sprague Dawley (Indianapolis, IN) and randomly assigned to one of four experimental groups $(n=7 /$ group). Rats were used in accordance with guidelines set forth by the All-University Committee on Animal Use and Care at Michigan State University, an AAALAC accredited institution. Animals were housed with two per polycarbonate box and had free access to tap water and food (Tek Lad 22/5 Rodent Diet W 8649, Harlan, Indianapolis, IN).

Treatment Protocols-Rats were instilled intranasally (IN) with $150 \mu$ l volumes of endotoxin (LPS; lipopolysaccharide from Pseudomonas aeruginosa, Sigma St. Louis, MO) in pyrogen-free saline into each nasal passage (300 $\mu$ l total volume), for total doses of 0 or $20 \mu \mathrm{g}$. For instillations, rats were lightly anesthetized with $4 \%$ isoflurane in oxygen. A second instillation was given $24 \mathrm{~h}$ later. IN challenge procedures were conducted between 10:00 and 11:00 am each day. Two days prior to IN LPS procedures, rats were given a daily supplementation regimen of $30 \mathrm{mg} / \mathrm{kg}$ body weight of $\gamma$-tocopherol $(\gamma \mathrm{T} ; 96 \%$ pure by HPLC and NMR r,r,r isomer; Yasoo Health Inc., Johnson City, TN) prepared in tocopherolstripped corn oil (Dyets Inc., Bethlehem, PA). $\gamma \mathrm{T}$ was administered by oral gavage each day at 6:00pm for four consecutive days, such that two administrations of $\gamma \mathrm{T}$ were given before the first IN LPS dose (Figure 1A). Animals were euthanized and tissues were collected $24 \mathrm{~h}$ after the last dose of IN LPS.

Necropsy and Tissue Preparation-Animals were anesthetized with sodium pentobarbital $(50 \mathrm{mg} / \mathrm{kg})$ and euthanized by exsanguination. The trachea was cannulated and the heart and lung were excised en bloc. The bronchus to the left lung was temporarily clamped and $5 \mathrm{ml}$ of sterile saline was instilled through the tracheal cannula and withdrawn to recover bronchoalveolar lavage fluid (BALF) from the right lung lobes. A second saline lavage was performed and combined with the first.

After lavage, the left lobe was inflated under constant pressure $\left(30 \mathrm{~cm} \mathrm{H}_{2} \mathrm{O}\right)$ with neutralbuffered formalin for $2 \mathrm{~h}$, and twenty-four hours later, a $3 \mathrm{~mm}$ section was excised from the left lung lobe at the level of the fifth airway generation, embedded in paraffin, and 5-6 $\mu \mathrm{m}$ thick sections were cut from the anterior surface. PMNs within the lung sections were immunohistochemically identified by using a rabbit-derived antibody to rat PMNs (gift of Robert A. Roth, MSU), and stained with an alkaline phosphatase system (Vector Red Substrate Kit; Vector Labs, Burlingame, CA) to form a red stain as imaged under a light microscope.

Bronchoalveolar Lavage-Total leukocytes in BALF were enumerated with a hemocytometer, and fractions of eosinophils, neutrophils, macrophages, and lymphocytes were determined in a cytospin sample stained.

Morphometry of submucosal PMNs-The numeric density of neutrophils in the mucosa underlying the respiratory epithelium of conducting pulmonary airways, were determined by counting PMN-positive stained cells with distinct neutrophil morphology. The length of the basal lamina was calculated from its contour length in a digitized image 
and the data is expressed as the number of neutrophils divided by the length of the underlying basal lamina.

\section{HUMAN STUDIES}

Volunteer Recruitment and Inclusion Criteria-This protocol was reviewed and approved by the University of North Carolina Committee on the Rights of Human Subjects (Institutional Review Board). This study was also reviewed and approved by the US Food and Drug Administration (IND 13,004). The study was listed on clinicaltrials.gov, NCT00631085. This was a double blinded, placebo controlled crossover study comparing the effect of supplementation with a $\gamma$ T-enriched supplement on LPS-induced airway neutrophilia (Figure 3 ) in healthy volunteers with negative methacholine challenge. All subjects underwent a physical examination, routine blood panel with $\mathrm{CBC}$ and differential cell count, allergy skin testing, and methacholine challenge, as described by Hernandez et al. 2012 [7]. All subjects were screened for their ability to provide an adequate induced-sputum sample during their screening session, defined by $>250,000$ cells, $>50 \%$ viability, $<40 \%$ squamous cells.

Randomization-The randomization list was prepared by a biostatistician (Dr. Zhou) using SAS@ and provided to the UNC Investigational Drug Pharmacy. Only the pharmacist and the statistician had access to the randomization schedule. If a subject passed their baseline sputum screening session, the study staff contacted the pharmacy. The pharmacy then assigned a randomization number to each subject.

The seven day supply of study drug was dispensed in opaque bottles from the Investigational Drug Pharmacy to the study staff who directly observed doses 1, 6 and 7 taken by the volunteers at the CEMALB. Doses $2-5$ were provided to the volunteers, who received a daily phone call or email from the CEMALB study staff to confirm that they took the daily dose. Subjects were randomized to either orally administered $\gamma \mathrm{T}$ or placebo during the initial study period each day for 7 consecutive days, and received the alternative drug during the crossover period (Figure 3). There was a minimum washout period of 14 days between the placebo or active treatment periods. Both CEMALB study staff and subjects were blinded to the treatment regimens.

YT Supplementation-A $\gamma \mathrm{T}$-enriched supplement and a sunflower oil (placebo) were provided by YASOO Health Inc. (Johnson City, TN). Both gel capsules were identical in appearance and taste. Each $\gamma \mathrm{T}$-enriched geltab tablet was $830 \mathrm{mg}$ (lot number 14081) and was composed of $50 \mathrm{mg} \mathrm{d}$ - $\alpha$-tocopherol, $240 \mathrm{mg} \mathrm{d}-\beta$ tocopherol plus $\mathrm{d}-\delta$-tocopherol, and $540 \mathrm{mg} \mathrm{d}-\gamma$ tocopherol.. Subjects took 2 geltabs of either placebo or $\gamma \mathrm{T}$-enriched supplement.

Endotoxin Inhalation Challenge-Clinical Center Reference Endotoxin (CCRE), referred to as LPS, was provided by the National Institutes of Health Clinical Center. A dose of 20,000 endotoxin units (EU) was previously shown to induce sputum neutrophils, and was demonstrated to be safe and well tolerated in previous studies [7]. Subjects inhaled LPS via a DeVilbiss Ultraneb 99 ultrasonic nebulizer. Induced sputum was obtained 6 hours post CCRE inhalation and processed as previously described [13-16]. Cell viability (trypan blue exclusion) and total cell counts were assessed in a Neubauer hemacytometer and differential cell counts were performed on cytocentrifuged cells stained with a modified Wright's stain, (Hema-Stain -3, Fisher Scientific). 
Serum Analysis of Tocopherols and $\mathrm{y}$-CEHC- $-\gamma$-T and 5-nitro- $\gamma$-T were measured in the serum by a HPLC assay with electrochemical detection; $\gamma$-CEHC was analyzed using a HPLC assay with fluorescent detection, as described by Wiser et al 2008 [12].

Mediator Measurement-Cytokines from sputum supernatants were measured using multi-plex technology (Meso ScaleDiscovery/MSD, Gaithersburg, MD). Each sample was analyzed with the Human TH1/TH2 10-Plex Ultra Sensitive Kit. All supernatant samples were diluted 1:4 and had a final DTT concentration of $<1 \mathrm{mM}$ where no deleterious effects have been observed using the MSD platform.

Flow cytometry-Expression of selected cell surface molecules on sputum leukocytes was quantified via multicolor flow cytometry (FCM) using a BD LSR-II flow cytometer (BD Immunocytometry Systems; San Jose, CA). These included molecules associated with antigen presentation and specific immunity (CD86/B7.2, HLA-DR/MHC-II,), as well as innate immune function (CD11b/CR3, CD14/LPS receptor). C45 positive sputum leukocyte populations were identified and gated on the basis of light scatter properties and differential expression of CD45 (pan-leukocyte marker) and lineage specific surface markers as previously described [17]. Up-or down-regulation of specific surface molecules was quantified as a change in the mean fluorescent intensity (MFI) of the gated population. Fluorochrome-labeled antibodies were obtained from BD Biosciences (CD11b-PE-CY5, CD45-APC-Cy7, HLA-DR-PerCP, Beckman-Coulter (CD14-APC, CD86-PE). Appropriate, nonspecific, labeled isotypic control antibodies were also obtained from these sources.

Sample Size Calculations-To estimate sample size, we used previously published data from our previous LPS inhalation studies [5]. LPS induces 66\% PMNs (of total cells) in sputum ( $\mathrm{SD}=19, \mathrm{n}=9)$, compared to baseline samples having a mean PMN content of $37 \%$ $(\mathrm{SD}=19)$. Assuming that $\gamma \mathrm{T}$-enriched therapy could completely inhibit the response to LPS (thus providing a treatment effect of $29 \%$ ), we calculated that 10 volunteers would provide an adequate sample (alpha $=0.05$, power $90 \%$ ) to compare the difference in PMN content while a subject is in the placebo arm versus the active arm. We inflated this sample size to 18 to account for screen failures for induced sputum measurements, and increased drop-out from crossover design challenge studies.

Statistical Analysis-For analysis of cellular responses as well as cytokine and cell surface marker changes within each group for the human studies, we compared the baseline responses to the post-LPS responses using Wilcoxon Ranked sum tests as the data were not normally distributed. Mean and standard error of the mean are presented in the text and in Table 2. For the rat studies, data were analyzed using a completely randomized analysis of variance. Multiple comparisons were made by Student-Newman-Keuls post hoc test. Criterion for significance was taken to be $p \leq 0.05$. Both human and rat data are expressed in graphs as mean \pm standard error of the mean (SEM).

To analyze the sputum cellularity, we used a repeated measures analysis of variance. We accounted for carryover effects by first testing for treatment and period effects using the graphical presentations described by Jones and Kenward (section 3, 2003).

We followed up the above graphic observations with a two sample t-test procedure to evaluate the significance level of the treatment, the period and the carryover effects. In the absence of significant carryover effect, the standard error term in the t-test is estimated using the combined data from both trial periods. Otherwise, the standard error is estimated using period 1 data only. 
We also used a linear mixed model approach that considers the above individual tests in a global, unified way where all data are used at the same time. This provided a more powerful analysis than the two sample t-tests, especially when there is a significant carryover effect. This approach was used for assessment of the percent neutrophil data, where a carryover effect was noted. The linear mixed model is formulated as follows:

$$
\begin{aligned}
& Y_{11 k}=\mu+\pi_{1}+\tau_{1}+s_{1 k}+\varepsilon_{11 k} ; Y_{12 k}=\mu+\pi_{2}+\tau_{2}+\lambda_{1}+s_{1 k}+\varepsilon_{12 k} ; \\
& Y_{21 k}=\mu+\pi_{1}+\tau_{2}+s_{2 k}+\varepsilon_{21 k} ; Y_{22 k}=\mu+\pi_{2}+\tau_{1}+\lambda_{2}+s_{2 k}+\varepsilon_{22 k},
\end{aligned}
$$

Where $\mu$ is grand mean. $\pi_{1}$ and $\pi_{2}$ are period effects. $\tau_{1}$ and $\tau_{2}$ are treatment effects. $\lambda_{1}$ and $\lambda_{2}$ are carryover effects for different sequences. $\varsigma_{1 \kappa}$ and $\varsigma_{2 \kappa}$ are individual random effects within each sequence. $\varepsilon$ 's are random errors. Subscript $k$ is for subjects. SAS PROC MIXED is used. In most cases, the results from two sample t-tests and linear mixed models are consistent.

\section{Results}

\section{Effect of YT on LPS-induced airway cellularity in rats}

LPS induced significant neutrophil accumulation in the BALF 24h after the last instillation (Figure 1b). In rats given oral $\gamma$ T supplementation, LPS-induced BALF neutrophil numbers were reduced to the levels similar to control rats. To evaluate if $\gamma \mathrm{T}$ treatment affected neutrophil recruitment into the airway lumen versus tissue infiltration, we also evaluated neutrophil accumulation in the peribronchial space (generation 5 rat bronchi), quantified as number of submucosal neutrophils/mm of basal laminae. LPS challenge caused significant recruitment of neutrophils into alveolar parenchyma and the submucosa surrounding conducting airways that was attenuated by $\gamma \mathrm{T}$ treatment (Figures 1c, 2). These data demonstrate that $\gamma \mathrm{T}$ mitigates both the tissue infiltration and accumulation in airways of neutrophils that are elicited by challenge with LPS.

\section{Subject Demographics}

Eighteen healthy volunteers were enrolled. Four subjects were excluded after failing the induced sputum screening. One subject was excluded after randomization but before receiving the first dose of study drug due to an exacerbation of an underlying medical disorder. Thirteen subjects successfully completed the study (Table 1). After screening, each subject completed the entire protocol over a period of no greater than 12 weeks. Four subjects received active treatment during period 1; nine subjects received placebo treatment during period 1 . The washout period varied from 2 weeks to 8 weeks.

Only one subject was atopic, with sensitization to grass. He completed his study procedures during the months of December and January. All subjects tolerated the inhaled LPS challenge and supplementation without significant changes in vital signs, symptom scores, or decrements in spirometry (data not shown).

\section{Effect of $\mathbf{Y}^{\mathrm{T}}$ supplementation on serum tocopherol levels}

Serum levels of $\gamma \mathrm{T}$, its primary metabolite $\gamma$ CEHC, and its nitrated product 5-nitro- $\gamma \mathrm{T}$ were determined immediately before the inhaled LPS challenge during the active and placebo treatment periods (i.e. 6 hour timepoint), and 24 hours after the last dose of the active or placebo supplement. Subjects had already consumed 1 week of either placebo or active treatment prior to these assessments. We found significantly increased levels of $\gamma \mathrm{T}$ and $\gamma$ CEHC during the $\gamma \mathrm{T}$ supplement period compared to placebo for both these timepoints (Figure $4 a-b$ ). We found significantly decreased serum 5-nitro- $\gamma \mathrm{T}$ during the active 
treatment period compared to placebo (Figure 4c), suggesting decreased nitrosative stress during the $\gamma \mathrm{T}$ supplementation period.

\section{Effect of YT on LPS-induced Peripheral Blood Leukocytes}

LPS inhalation similarly and significantly increased the peripheral blood white blood cell count and the absolute neutrophil count during the placebo and $\gamma$ T supplementation periods (Figure 5a-b). There was no increase in peripheral blood eosinophil count during either treatment phase after LPS inhalation.

\section{Effect of YT on LPS-induced Sputum Cellularity}

Inhaled LPS significantly increased induced sputum \%neutrophils during the placebo period $(17.9 \%, \pm 3.5 \%, \mathrm{p}<0.01)$, but not during the active treatment period $(7.4 \%, \pm 8.6)$. Induced sputum cell percentages for each treatment period are provided in Table 2. While there was minimal change in sputum \%eosinophil counts after LPS challenge during the $\gamma \mathrm{T}$ treatment period, inhaled LPS increased sputum \%eosinophil count during the placebo period, approaching significance $(\mathrm{p}=0.06)$. We detected a carryover effect for the \%neutrophils in sputum (i.e. increased \%neutrophils at baseline for period 2, greater for the sequence Placebo-Active than for Active-Placebo), but not for induced sputum \%eosinophils. After accounting for both period effect and carryover effect in the statistical analysis, we found that $\gamma \mathrm{T}$ supplementation significantly reduced the \%neutrophils and \%eosinophils in sputum after LPS challenge (Figure 5c-d).

\section{Effect of $y^{\top}$ on sputum cytokine and chemokine levels}

We assayed sputum supernatants $(\mathrm{N}=13)$ for various $\mathrm{TH} 1$ and $\mathrm{TH} 2$ cytokines as exploratory measures of how this $\gamma \mathrm{T}$ intervention may have worked in this model of LPS-induced airway inflammation. Induced sputum supernatants were assessed for the TH1 cytokines IL-1 $\beta$, IL-6, and TNF-a (Table 2). There was a significant increase after inhaled LPS challenge in IL- $1 \beta$ during the placebo treatment period ( $\mathrm{p}=0.04)$, but not during the active period ( $\mathrm{p}=0.6$ ). There were no significant changes in IL-6 or TNF- $\mathrm{a}$ with inhaled LPS challenge during the placebo or the active treatment periods.

\section{Effect of yT on sputum leukocyte surface markers}

Induced sputum macrophages and neutrophils were assayed for surface markers consistent with LPS-stimulation such as TLR4 and CD14, and for markers consistent with granulocyte chemotaxis (neutrophil CD11b). Nine pairs of sputum samples were available for analysis. We found similar and significant upregulation of CD14 on induced sputum monocytes during the active $(\mathrm{p}=0.01)$ and placebo $(\mathrm{p}=0.004)$ treatment periods (data not shown). We found non-significant increased expression of macrophage TLR4 and neutrophil CD11b during the placebo or active treatment periods (data not shown). There was no detectable effect with $\gamma \mathrm{T}$ treatment.

\section{Discussion}

The purpose of the pre-clinical and early phase human studies were to determine if in vivo $\gamma \mathrm{T}$ supplementation could be an effective intervention in a model of neutrophilic airway inflammation induced by LPS inhalation. Results from the rat studies suggest that $\gamma \mathrm{T}$ supplementation interferes with generalized neutrophil recruitment to the lung, with LPSelicited neutrophil accumulation in both tissue and airspaces reduced to control levels by $\gamma \mathrm{T}$. In healthy volunteers, we found that $\gamma \mathrm{T}$ supplementation decreased LPS-induced $\%$ neutrophils in sputum by half. Based on the rodent data, we suspect that decreased sputum neutrophil counts were due to $\gamma \mathrm{T}$ effects on neutrophil recruitment to the lung tissue, and not 
on interference with neutrophil transmigration across the epithelial cell barrier and into lung airspaces.

Investigators from our group have previously shown that $\gamma \mathrm{T}$ effectively inhibits COX-2 activity in LPS-stimulated macrophages and IL-1 $\beta$-stimulated epithelial cells as well as cytokine production in animal models of inflammation $[18,19]$ independently of its action as an antioxidant. These actions appear to be partially mediated primarily by $\gamma$-CEHC, the terminal metabolite of $\gamma \mathrm{T}$. In addition, $\gamma \mathrm{T}$ has been found to reduce calcium ionophoreinduced production of the neutrophil chemoattractant LTB4 by neutrophil-like differentiated HL-60 cells and LTC4 production by eosinophil-like clone 15 HL-60 cells [20]. Wiser et al. [12] previously found that peripheral blood monocyte release of pro-inflammatory cytokines TNF-a, IL-6, and IL-1 $\beta$ after ex vivo LPS stimulation were significantly reduced after subjects took one week of a $\gamma$ T-enriched supplement. The supplement used by Wiser et al. [12] provided comparable serum levels of $\gamma \mathrm{T}$ and $\gamma \mathrm{CEHC}$ to the supplement used in this study.

As exploratory measures, we assessed pro-inflammatory cytokine levels in induced sputum, as well as surface expression of innate immune molecules (CD14, TLR4, CD11b) shown to be upregulated with inhaled LPS challenge. We found that IL-1 $\beta$ in induced sputum was significantly increased during the placebo treatment period, but not during the active treatment period. Based on the ex vivo data provided by Wiser et al, in conjunction with our exploratory analysis, we speculate that interference with pro-inflammatory cytokine production during the active period may have impacted the neutrophil response, but this would have to be tested more directly with a study powered to examine that association. Although we detected some LPS-induced changes in sputum leukocyte CD14, TLR4, and CD11b, we did not detect a $\gamma \mathrm{T}$ treatment effect. However, it is unclear whether this is due to lack of $\gamma \mathrm{T}$ action on these molecules, or whether this may have been secondary to the limited sample size available for flow cytometric analysis.

Surprisingly, we found that LPS inhalation increased sputum eosinophils in this mostly nonatopic population, and that $\gamma \mathrm{T}$ treatment blunted this effect. LPS challenge has been shown to recruit eosinophils into the pleural cavities of mice injected with LPS, and this effect appears to be dependent on airway macrophages and T lymphocytes [21, 22], independent of IL-5. $\gamma \mathrm{T}$ was found to inhibit IL-13 activated eotaxin-3 secretion in the lung epithelial cell line A549 [23], suggesting a mechanism by which eosinophil recruitment can be blunted by $\gamma \mathrm{T}$ supplementation. In a rodent model of allergic rhinitis and asthma using ovalbumin (Ova) as a test allergen, we found that daily administration of $\gamma \mathrm{T}$ prior to Ova challenge depressed eosinophil infiltration into the airways, as well as production of nasal $\mathrm{TH} 2$ cytokines in the nasal airway and pulmonary eicosanoids [24]. $\gamma \mathrm{T}$ effects on eosinophil recruitment should ideally be tested in in vivo human models of allergic airway inflammation to confirm such effects.

This early phase study has numerous limitations. An ideal crossover study design should have no carryover or period effects in order to more accurately interpret a treatment effect. We found a carryover effect related to the primary endpoint, percent neutrophils in induced sputum. This was surprising, but possibly explained by the fact that the length between periods varied from 2 weeks to 8 weeks. In a time-course study of repeated endotoxin inhalation challenges with a much higher dose of endotoxin, Doyen et al found that a period of 7 days was sufficient to prevent pro-inflammatory carryover [25]. Although the reason for the sputum carryover effect in our study is unclear, future studies should have more uniform washout periods to minimize variability. The randomization sequence did not generate relatively equal numbers receiving either placebo or active treatment during the first period of the study, possibly contributing to the carryover effect. Our study assessed the effect of 
$\gamma$ T supplementation 6 hours after inhaled LPS, previously found by others to have maximal $\%$ neutrophils in sputum [25]. Examining the effects of this intervention on neutrophil clearance at later timepoints would be of interest for therapeutics targeting persistent airway neutrophils in conditions such as COPD and neutrophil-predominant asthma. In a pilot study of a supplement that contained $\gamma \mathrm{T}$ (among other antioxidant compounds), 7 patients with cystic fibrosis had reduced numbers of induced sputum neutrophils after 4 weeks of daily supplementation [26]. Finally, we did not design the study to examine epithelial-cell mediated signaling, such as evidence of eotaxin-3 secretion, that is difficult to detect in induced sputum samples. Further work examining the in vivo effects of $\gamma \mathrm{T}$ on eotaxin-3 would have to sample epithelial cells as a primary endpoint.

\section{Conclusions}

In conclusion, we found that $\gamma \mathrm{T}$ supplementation reduced neutrophil recruitment to the airway in both a rat model and in an early phase IIa study with healthy volunteers. We detected decreased levels of 5-nitro- $\gamma \mathrm{T}$ during the active treatment period. 5-nitro- $\gamma \mathrm{T}$ is recognized to be a marker of nitrosative stress, and is often elevated during systemic inflammation [27]. Supplementation with $\gamma \mathrm{T}$ may be beneficial as part of the therapeutic strategy in airway diseases characterized by airway neutrophilic inflammation, such as COPD and neutrophil-predominant asthma.

\section{Acknowledgments}

Funding Sources: MLH is supported by NIEHS K23-ES021745; KM, HBW, JCL, NEA, QJ, HZ, and DBP are supported by NIAID U19AI077437. JGW is supported by EPA R834797. QJ is supported by NCCAM 5 R01AT006882. This work was also supported by P01AT002620 from the National Center for Complementary and Alternative Medicine.

\section{Abbreviations}

\begin{tabular}{|c|c|}
\hline LPS & Lipopolysaccharide \\
\hline$\gamma \mathbf{T}$ & gamma-tocopherol \\
\hline$\gamma$-CEHC & 2, 7, 8-trimethyl-2S-( $\gamma$-carboxyethyl)-6-hydroxychromane \\
\hline LTB4 & Leukotriene B4 \\
\hline BALF & Bronchoalveolar Lavage Fluid \\
\hline \%PMNS & \%neutrophils \\
\hline WBC & White Blood Cell \\
\hline ANC & Absolute Neutrophil Count \\
\hline IN & intranasal \\
\hline COPD & Chronic Obstructive Pulmonary Disease \\
\hline
\end{tabular}

\section{References}

1. Gern JE, Busse WW. Relationship of viral infections to wheezing illnesses and asthma. Nat Rev Immunol. 2002; 2(2):132-138. [PubMed: 11910895]

2. Peden DB. The epidemiology and genetics of asthma risk associated with air pollution. J Allergy Clin Immunol. 2005; 115(2):213-219. quiz 220. [PubMed: 15696070]

3. Reed CE, Milton DK. Endotoxin-stimulated innate immunity: A contributing factor for asthma. J Allergy Clin Immunol. 2001; 108(2):157-166. [PubMed: 11496229] 
4. Alexis NE, et al. Development of an inhaled endotoxin challenge protocol for characterizing evoked cell surface phenotype and genomic responses of airway cells in allergic individuals. Ann Allergy Asthma Immunol. 2008; 100(3):206-215. [PubMed: 18426139]

5. Alexis NE, et al. Acute LPS inhalation in healthy volunteers induces dendritic cell maturation in vivo. J Allergy Clin Immunol. 2005; 115(2):345-350. [PubMed: 15696093]

6. Hernandez ML, et al. Comparative airway inflammatory response of normal volunteers to ozone and lipopolysaccharide challenge. Inhal Toxicol. 2010; 22(8):648-656. [PubMed: 20540623]

7. Hernandez ML, et al. Atopic asthmatic patients have reduced airway inflammatory cell recruitment after inhaled endotoxin challenge compared with healthy volunteers. The Journal of allergy and clinical immunology. 2012; 130(4):869 e2-876 e2. [PubMed: 22770265]

8. Alcorn JF, Crowe CR, Kolls JK. TH17 cells in asthma and COPD. Annual review of physiology. 2010; 72:495-516.

9. Jiang Q, Ames BN. Gamma-tocopherol, but not alpha-tocopherol, decreases proinflammatory eicosanoids and inflammation damage in rats. FASEB journal : official publication of the Federation of American Societies for Experimental Biology. 2003; 17(8):816-822. [PubMed: 12724340]

10. Jiang Q, et al. gamma-tocopherol and its major metabolite, in contrast to alphatocopherol, inhibit cyclooxygenase activity in macrophages and epithelial cells. Proceedings of the National Academy of Sciences of the United States of America. 2000; 97(21):11494-11499. [PubMed: 11005841]

11. Jiang Q, et al. Long-chain carboxychromanols, metabolites of vitamin E, are potent inhibitors of cyclooxygenases. Proceedings of the National Academy of Sciences of the United States of America. 2008; 105(51):20464-20469. [PubMed: 19074288]

12. Wiser J, et al. In vivo gamma-tocopherol supplementation decreases systemic oxidative stress and cytokine responses of human monocytes in normal and asthmatic subjects. Free Radic Biol Med. 2008; 45(1):40-49. [PubMed: 18405673]

13. Alexis NE, et al. The glutathione-S-transferase Mu 1 null genotype modulates ozone induced airway inflammation in human subjects. J Allergy Clin Immunol. 2009; 124(6):1222 e5-1228 e5. [PubMed: 19796798]

14. Lay JC, et al. Ozone enhances markers of innate immunity and antigen presentation on airway monocytes in healthy individuals. J Allergy Clin Immunol. 2007; 120(3):719-722. [PubMed: 17586033]

15. Alexis NE, Eldridge MW, Peden DB. Effect of inhaled endotoxin on airway and circulating inflammatory cell phagocytosis and CD11b expression in atopic asthmatic subjects. J Allergy Clin Immunol. 2003; 112(2):353-361. [PubMed: 12897742]

16. Alexis NE, et al. Biological material on inhaled coarse fraction particulate matter activates airway phagocytes in vivo in healthy volunteers. J Allergy Clin Immunol. 2006; 117(6):1396-1403. [PubMed: 16751003]

17. Lay JC, Peden DB, Alexis NE. Flow cytometry of sputum: assessing inflammation and immune response elements in the bronchial airways. Inhalation toxicology. 2011; 23(7):392-406.

[PubMed: 21639708]

18. Jiang Q, et al. gamma-tocopherol and its major metabolite, in contrast to alphatocopherol, inhibit cyclooxygenase activity in macrophages and epithelial cells. Proc Natl Acad Sci U S A. 2000; 97(21):11494-11499. [PubMed: 11005841]

19. Jiang Q, et al. Gamma-tocopherol supplementation inhibits protein nitration and ascorbate oxidation in rats with inflammation. Free Radic Biol Med. 2002; 33(11):1534-1542. [PubMed: 12446211]

20. Jiang Z, Yin X, Jiang Q. Natural forms of vitamin E and 13'-carboxychromanol, a long-chain vitamin $\mathrm{E}$ metabolite, inhibit leukotriene generation from stimulated neutrophils by blocking calcium influx and suppressing 5-lipoxygenase activity, respectively. Journal of immunology. 2011; 186(2):1173-1179.

21. Bozza PT, et al. Requirement for lymphocytes and resident macrophages in LPS induced pleural eosinophil accumulation. J Leukoc Biol. 1994; 56(2):151-158. [PubMed: 8071592] 
22. Penido $\mathrm{C}$, et al. The role of gammadelta $\mathrm{T}$ lymphocytes in lipopolysaccharide-induced eosinophil accumulation into the mouse pleural cavity. J Immunol. 1997; 159(2):853-860. [PubMed: 9218604]

23. Wang Y, et al. Vitamin E forms inhibit IL-13/STAT6-induced eotaxin-3 secretion by up regulation of PAR4, an endogenous inhibitor of atypical PKC in human lung epithelial cells. The Journal of nutritional biochemistry. 2012; 23(6):602-608. [PubMed: 21764283]

24. Wagner JG, et al. Gamma-tocopherol prevents airway eosinophilia and mucous cell hyperplasia in experimentally induced allergic rhinitis and asthma. Clin Exp Allergy. 2008; 38(3):501-511. [PubMed: 17970781]

25. Doyen V, et al. Time course of endotoxin-induced airways' inflammation in healthy subjects. Inflammation. 2012; 35(1):33-38. [PubMed: 21207124]

26. Papas KA, et al. A pilot study on the safety and efficacy of a novel antioxidant rich formulation in patients with cystic fibrosis. Journal of cystic fibrosis : official journal of the European Cystic Fibrosis Society. 2008; 7(1):60-67. [PubMed: 17569601]

27. Christen S, et al. Analysis of plasma tocopherols alpha, gamma, and 5-nitro-gamma in rats with inflammation by HPLC coulometric detection. Journal of lipid research. 2002; 43(11):1978-1985. [PubMed: 12401897] 


\section{Highlights}

- Treatment options for neutrophilic airway inflammation are limited

- Inhaled LPS challenge is used to study neutrophil-predominant airway inflammation

- Gamma Tocopherol, $\gamma \mathrm{T}$, is a candidate targeting neutrophilic inflammation

- Oral $\gamma \mathrm{T}$ supplementation reduced LPS-induced airway neutrophils in rats and humans 

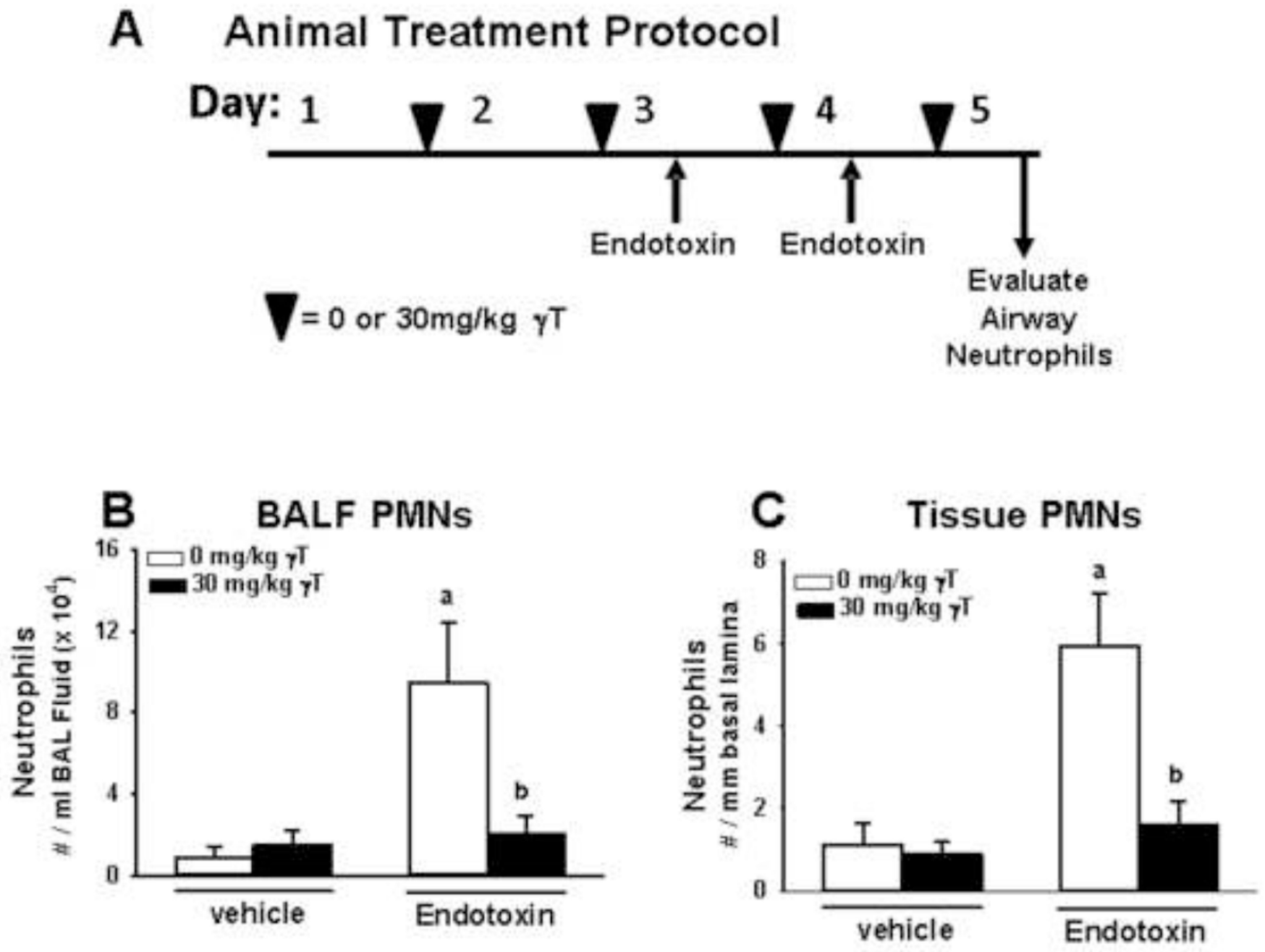

a - significantly different from respective group treated with vehicle; $\rho<0.05$

b - significantly different from res pective group treated with $0 \mathrm{mg} / \mathrm{kg} \gamma \mathrm{T} ; \rho<0.05$

Figure 1. Pre-clinical studies using rats

A) Time Course of treatment with $\gamma$ T (black triangles) and intranasal LPS (up-arrows). Treatment related effects of LPS and $\gamma \mathrm{T}$ on B) BALF and C) tissue PMNs collected 24h after the last LPS challenge. 


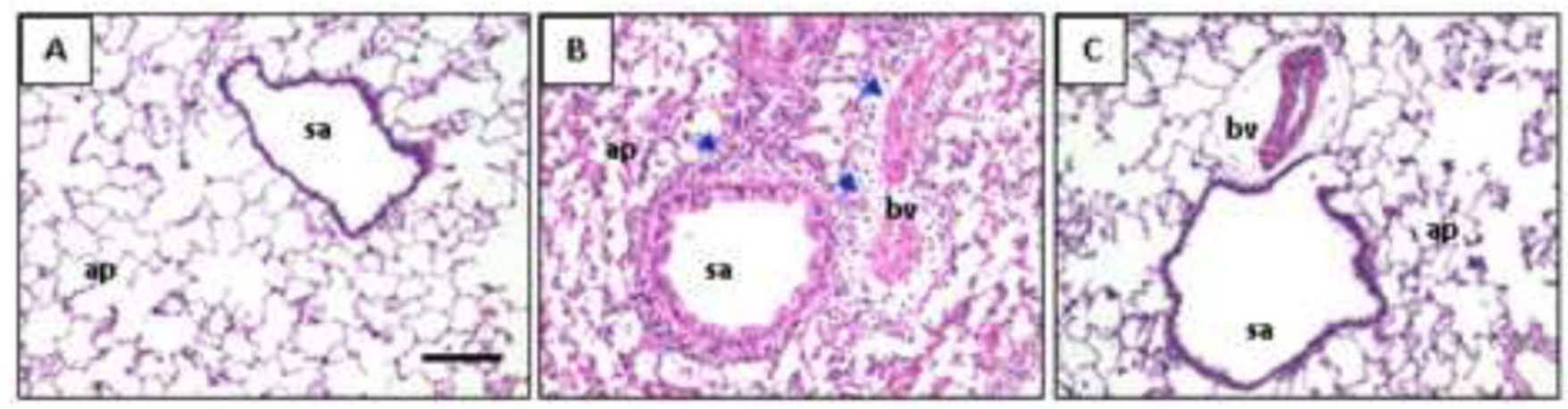

Figure 2. Lung histology in rats

Photomicrographs of lung tissues stained with hematoxylin \&eosin from rats after airway treatment with A) saline, B) endotoxin or C) endotoxin and $\gamma \mathrm{T}$. ap=alveolar parenchyma; bv = blood vessel; sa = small airway; arrows indicate inflammatory cell infiltrates.

Magnification bar $=20 \mathrm{~mm}$. 


\section{Phase lla Human Study Design}

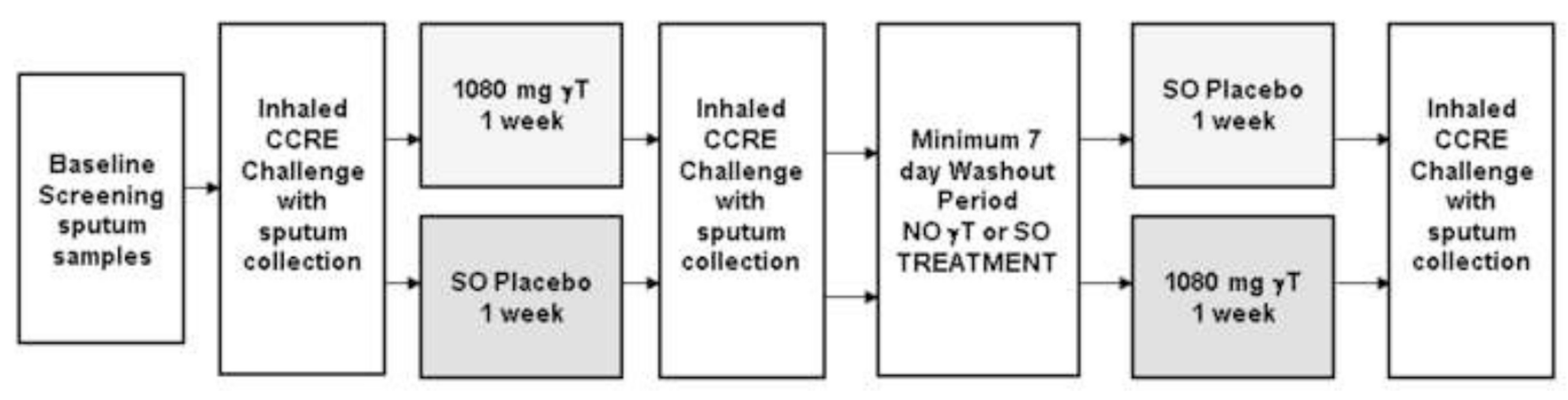

Figure 3. Phase IIa study design in healthy human volunteers

Randomized, placebo controlled crossover study of a $\gamma$ T-enriched supplement or sunflower oil placebo in 13 healthy volunteers. Subjects were challenged with 20,000 endotoxin units of Clinical Center Reference Endotoxin (CCRE), followed by sputum induction 6 hours after the challenge. 


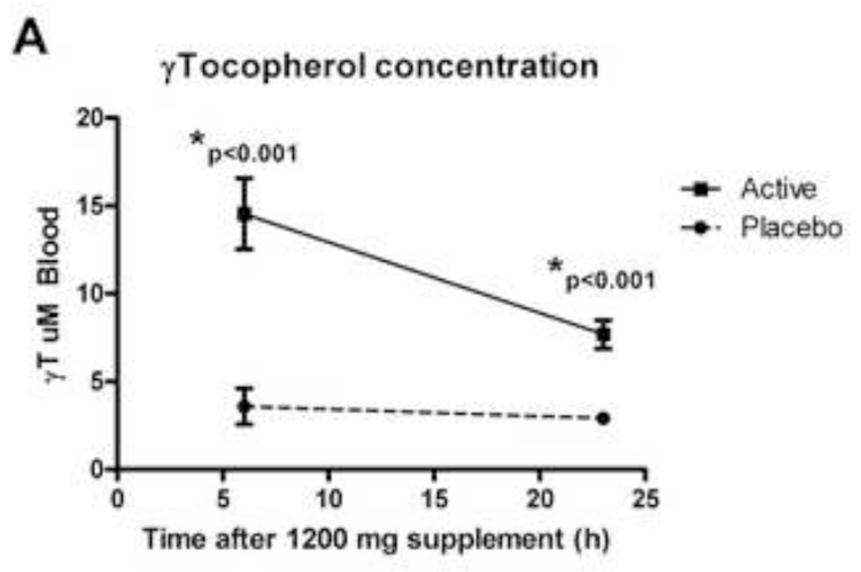

B
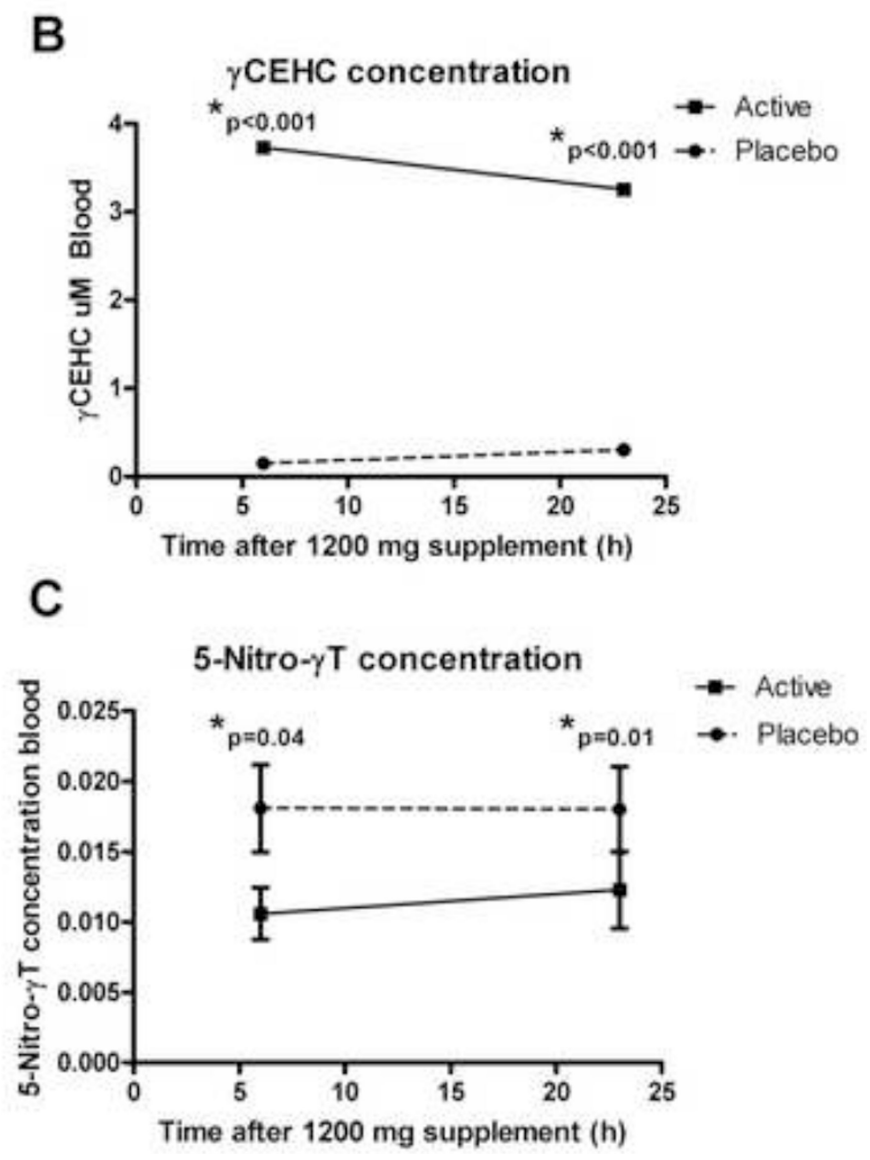

Figure 4. Serum levels of Tocopherols and $\gamma$-CEHC during the placebo and active study periods A). serum $\gamma \mathrm{T}$ concentrations B). serum $\gamma$-CEHC concentrations C). serum 5-nitro- $\gamma \mathrm{T}$ concentrations. 


\section{Blood}

A

B

White Blood Cell Count

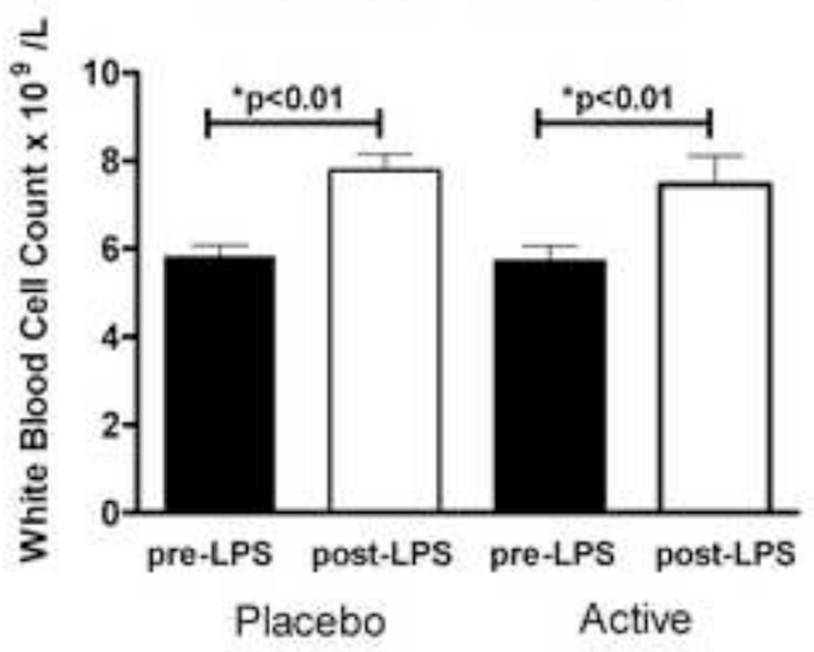

Blood PMNs

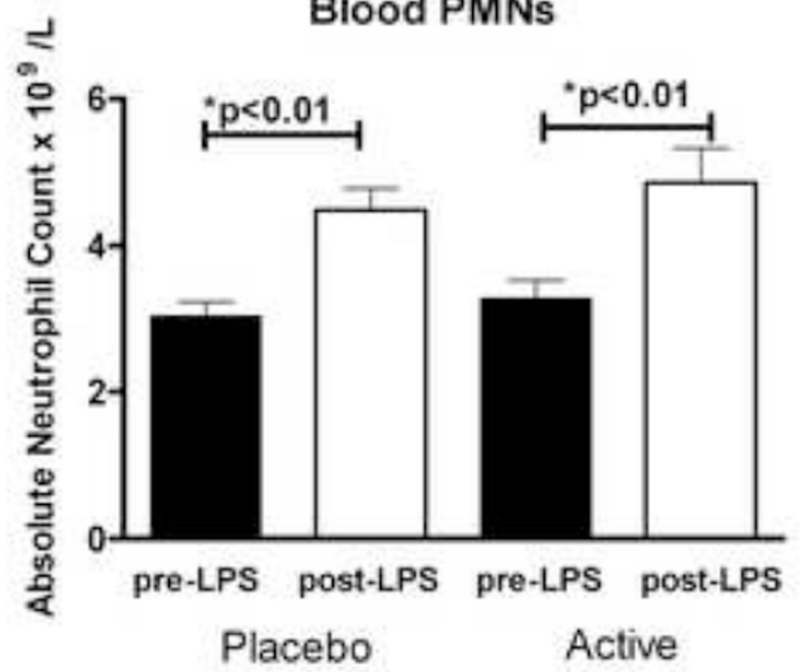

\section{Induced Sputum}
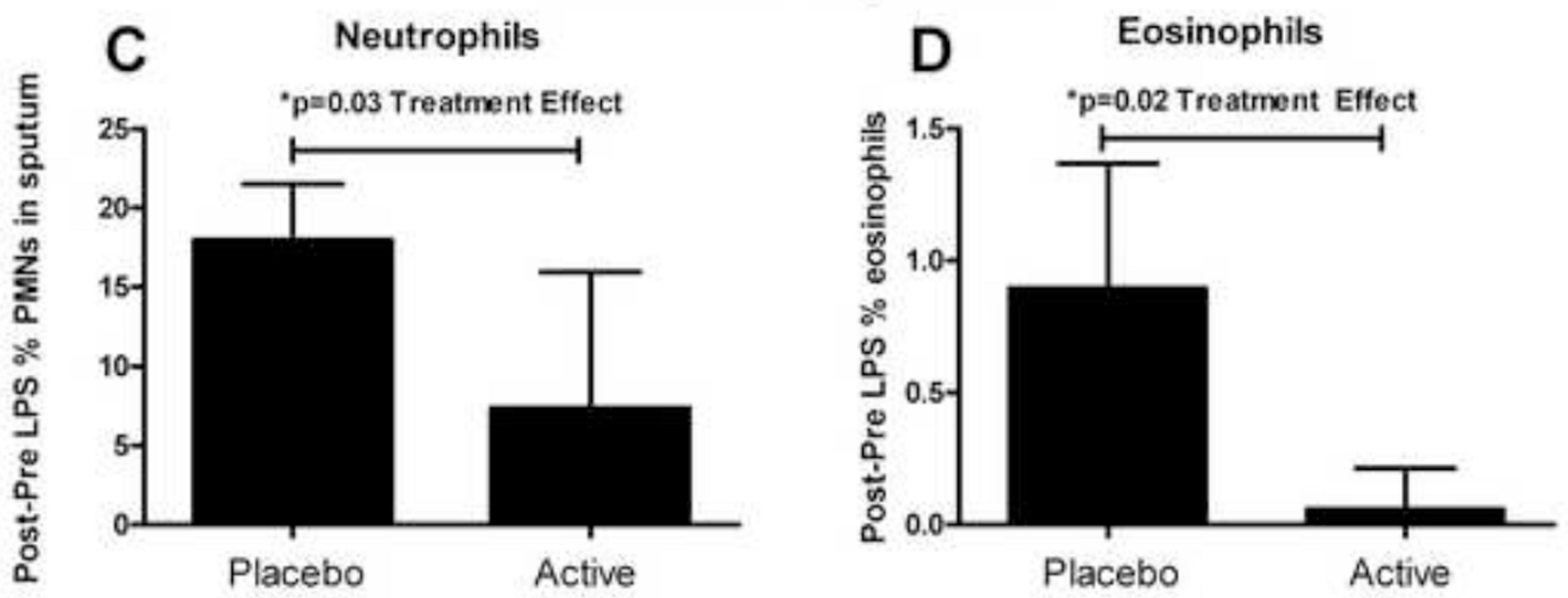

Figure 5. Blood and induced sputum leukocytes

Blood A). Total white blood cell (WBC) count B). Absolute neutrophil count (ANC). Induced sputum C). \%neutrophils (\%PMNS) and D). \%eosinophils. Values were calculated by subtracting the baseline (pre-LPS) \%PMN or \%eosinophil value to the post-LPS value to assess the deltas. Repeated measures ANOVA was used to calculate the treatment effect. 
Table I

Characteristics of healthy volunteers

\begin{tabular}{|c|c|}
\hline Clinical Characteristic & \\
\hline $\mathbf{N}$ & 13 \\
\hline Age (y), mean (SD) & $26 \pm 7$ \\
\hline Gender & $8 \mathrm{~F} / 5 \mathrm{M}$ \\
\hline Race & $\begin{array}{c}10 \text { Caucasian } \\
\text { African American } \\
1 \text { Asian }\end{array}$ \\
\hline BMI & $24.2 \pm 3.9$ \\
\hline Atopy & 1 (Grass) \\
\hline
\end{tabular}




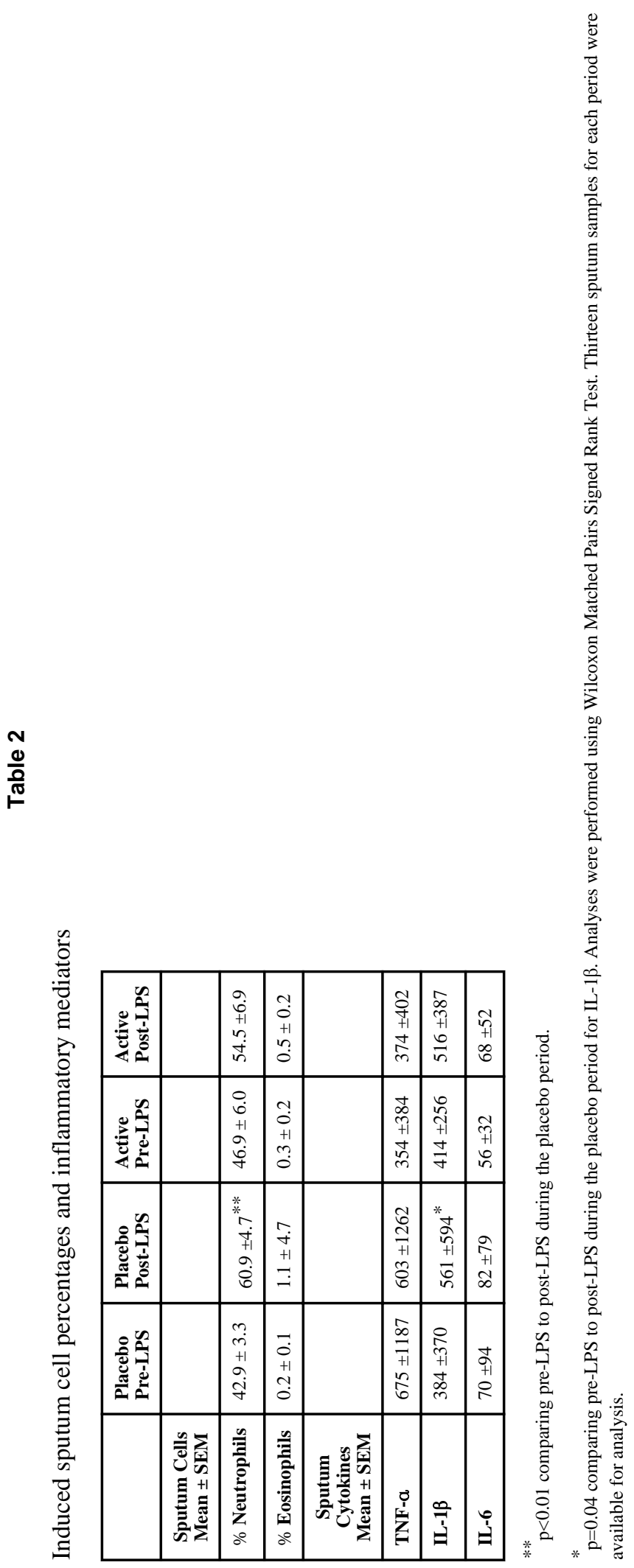

Free Radic Biol Med. Author manuscript; available in PMC 2014 July 01. 